\title{
LA RESPUESTA SOCIO-EMOCIONAL EN TIEMPOS DE PANDEMIA DE COVID 19 EN EL AULA DE SEGUNDO CURSO DE EDUCACIÓN PRIMARIA
}

\author{
Ángela Salueña Tena \\ Universidad de Zaragoza \\ José Luis Antoñanzas Laborda \\ Universidad de Zaragoza \\ jlantona@unizar.es \\ Laura Mateo Hernando \\ Universidad de Zaragoza
}

Recepción Artículo: 13 octubre 2021

Admisión Evaluación: 13 octubre 2021

Informe Evaluador 1: 13 octubre 2021

Informe Evaluador 2: 14 octubre 2021

Aprobación Publicación: 14 octubre 2021

\section{RESUMEN}

Las consecuencias provocadas por la pandemia COVID-19, han generado un deterioro en el desarrollo socioemocional de la población, afectando en gran medida a los niños. Esto se debe al aislamiento social que produce respuestas negativas en la expresión de las emociones, sentimientos y pensamientos, fomentando las relaciones sociales desde una perspectiva que no incluya el contacto físico. Por esta razón, a través de la propuesta de intervención planteada desde el ámbito educativo, se han desarrollado nuevas alternativas que mejoren la respuesta socioemocional infantil a través de actividades que impulsan las relaciones sociales a través del lenguaje corporal y la expresión emocional mediante el dibujo.

Palabras clave: desarrollo socioemocional; emociones; relaciones sociales; educación emocional y COVID-19

\footnotetext{
ABSTRACT

The social-emotional response in times of covid 19 pandemic in the second grade primary school classroom. The consequences caused by the COVID-19 pandemic have generated a deterioration in the socio-emotional development of people, mostly affecting children. This is due to social isolation that produces negative responses in the expression of emotions, feelings and thoughts, promoting social interactions that do not include physical contact. For this reason, through the intervention proposal raised by the educational field, new alternatives have been developed that improve the child's socio-emotional responses thanks to activities that promote social interaction using body language and emotional expressions through drawing.

Keywords: socio-emotional development; emotions; social relationships; emotional education and COVID $-19$
} 


\section{LA RESPUESTA SOCIO-EMOCIONAL EN TIEMPOS DE PANDEMIA DE COVID 19 EN EL AULA DE SEGUNDO CURSO DE EDUCACIÓN PRIMARIA}

\section{INTRODUCCIÓN}

La pandemia ha conllevado un deterioro de la expresión de las emociones y el desarrollo de las relaciones sociales creando una gran incidencia en el ámbito escolar. Por un lado, las emociones son definidas por Bisquerra (2003) con el concepto de autorregulación emocional, entendida como la capacidad que posee el niño de tolerar la rabia y la frustración, provocando una mejora en las habilidades sociales y la relación con su entorno.

Para ser más específicos, García (2020) afirma una necesidad de cambio en el sistema educativo que permite adaptar la nueva realidad social a la que los niños se enfrentan. Desde el punto de vista del docente, y según las aportaciones de Bisquerra (2002) en su investigación sobre las emociones, el maestro debe mantener una postura que empatice con el alumnado, mantener una comunicación entre el docente y el alumno, interesándose por los pensamientos y sentimientos del alumnado. Por lo tanto, la expresión de las emociones en el aula promueve una respuesta socioemocional del niño que ha sido perjudicada desde el comienzo de la COVID-19. Mi investigación ha girado en torno al segundo curso de Primaria, analizando una propuesta de mejora a la problemática socioemocional existente, Ilevándola a la práctica en una intervención en un aula.

\section{DESARROLLO SOCIOEMOCIONAL INFANTIL}

El objetivo más importante como docente es hacer de guía en el proceso de aprendizaje cognitivo y emocional, cuidando ambos aspectos como elementos clave en el crecimiento del alumno. El desarrollo socioemocional del niño hace referencia a un proceso interno, en una relación consigo mismo, y también a un proceso de externo de relaciones con los demás, distinguiendo sus emociones, y creando pautas emocionales y sociales de mayor complejidad. Bayley (2006). En resumen, Arrellano-Vaca (2021) asume el desarrollo socioemocional del niño como el transcurso para la adquisición de habilidades que comienza desde los primeros años de vida del niño abarcando la vida adulta.

\section{DESARROLLO SOCIOEMIOCIONAL A TRAVÉS DEL CONSTRUCTIVISMO SEGÚN JEAN PIAGET}

El estudio sobre el desarrollo socioemocional del niño puede entenderse desde la perspectiva del constructivismo que plantea Jean Piaget en su Teoría del Desarrollo Cognoscitivo. En el ámbito educativo, el constructivismo establece que las personas desarrollan sus capacidades cognitivas a través de interacciones. Por tanto, este paradigma se caracteriza por la participación de la persona siendo el protagonista de su propio proceso de conocimiento. Como su nombre indica, Munari (1994) apunta que el constructivismo propuesto por Piaget constituye una construcción de la propia persona adaptándose al contexto y entorno que le rodea, adaptando los conocimientos previos que ya posee y construyendo los nuevos que el entorno le puede proporcionar.

\section{CONCEPTO DE EMOCIONES}

El término de emoción abre un hilo de investigación numeroso que trabaja muchos aspectos, desde la medicina hasta la educación. La definición de emociones abre un abanico de términos sin delimitar ni concretar, haciendo un paso por el tiempo hasta la actualidad. Bisquerra (2003) sintetiza en forma de esquema el concepto de emoción:

Figura 1. Concepto de emoción. Bisquerra (2003)

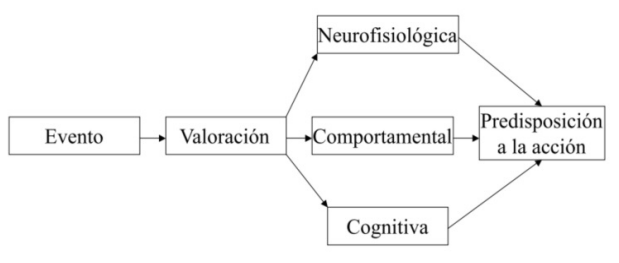


De modo general Adolphs (2019) define las emociones como estados funcionales que se encuentran en el cerebro aportando la causalidad de determinados comportamientos que van más allá de lo primitivo.

\section{ESTÍMULOS EMOCIONALES}

Adolphs (2019) distingue diferentes roles que regulan los estímulos emocionales:

Tabla 1. Múltiples roles para los llamados estímulos "emocionales". Adolphs (2019)

\begin{tabular}{|c|c|}
\hline $\begin{array}{l}\text { 1. Estímulo disparador del } \\
\text { circuito de supervivencia }\end{array}$ & Activa un circuito de supervivencia específico \\
\hline $\begin{array}{l}\text { Disparador innato } \\
\text { (incondicionado) }\end{array}$ & $\begin{array}{l}\text { Obtiene respuestas innatas a los estímulos sin la necesidad de } \\
\text { una exposición previa al estímulo y moviliza otros recursos } \\
\text { cerebrales para hacer frente a la oportunidad o desafío } \\
\text { presentado por el desencadenante innato. }\end{array}$ \\
\hline $\begin{array}{l}\text { Disparador aprendido } \\
\text { (condicionado) }\end{array}$ & $\begin{array}{l}\text { Potencialmente provoca respuestas innatas a los estímulos } \\
\text { después de estar asociado (a través del condicionamiento } \\
\text { pavloviano) con un desencadenante innato; De manera más } \\
\text { general, moviliza recursos cerebrales para hacer frente al } \\
\text { desafío u oportunidad señalada por el desencadenante } \\
\text { aprendido. }\end{array}$ \\
\hline 2. Incentivo & $\begin{array}{l}\text { Modula el comportamiento instrumental dirigido a objetivos } \\
\text { para ayudar a aprovechar la oportunidad o desafío señalado } \\
\text { por el estímulo que desencadena la activación de un circuito de } \\
\text { supervivencia específico. }\end{array}$ \\
\hline $\begin{array}{c}\text { Incentivo innato } \\
\text { (incondicionado o } \\
\text { primario) }\end{array}$ & $\begin{array}{l}\text { Aumenta el enfoque o la evitación del estímulo en un esfuerzo } \\
\text { por resolver el desafío o la oportunidad presente. }\end{array}$ \\
\hline $\begin{array}{l}\text { Incentivo aprendido } \\
\text { (condicionado o } \\
\text { secundario) }\end{array}$ & $\begin{array}{l}\text { Vigoriza y guía el comportamiento hacia situaciones en las que se } \\
\text { puede resolver el desafío o la oportunidad presente. }\end{array}$ \\
\hline 3. Reforzador & $\begin{array}{l}\text { Apoya el aprendizaje de asociaciones instrumentales o } \\
\text { pavlovianas }\end{array}$ \\
\hline $\begin{array}{c}\text { Refuerzo innato } \\
\text { (incondicionado o } \\
\text { primario) }\end{array}$ & $\begin{array}{l}\text { Induce la formación de asociaciones con estímulos neutros que } \\
\text { ocurren en su presencia (a través del condicionamiento } \\
\text { pavloviano) y a la formación de asociaciones con respuestas } \\
\text { que conducen a la presentación (estímulos apetitivos) o } \\
\text { eliminación (estímulos aversivos) del estímulo (a través del } \\
\text { condicionamiento instrumental). }\end{array}$ \\
\hline $\begin{array}{c}\text { Aprendidos } \\
\text { (condicionados o de } \\
\text { segundo orden) }\end{array}$ & $\begin{array}{l}\text { Induce la formación de asociaciones con otros estímulos (a } \\
\text { través del condicionamiento pavloviano de segundo orden) o } \\
\text { con respuestas dirigidas a objetivos (a través del } \\
\text { condicionamiento instrumental de segundo orden) }\end{array}$ \\
\hline
\end{tabular}




\section{LA RESPUEESTA SOCIO-EMOCIONAL EN TIEMPOS DE PANDEMIA DE COVID 19 EN EL AULA DE SEGUNDO CURSO DE EDUCACIÓN PRIMARIA}

\section{CONCEPTO DE COVID-19}

Velázquez (2020) expone desde la Academia de Ciencias de Cuba la grave situación que el mundo comenzó a lidiar contra ella en 2019 hasta la actualidad. El SARS-CoV-2, también llamado COVID-19 es un virus mayormente desconocido por la humanidad que ha causado 126604 fallecidos desde su aparición, afectando a toda la población, desde los jóvenes hasta la tercera edad causando problemas de mayor trascendencia en su estado de salud.

\section{REPERCURSIONES SOCIOEMOCIONALES DE LA PANDEMIA DE COVID-19 EN LAS RELACIONES SOCIALES INFANTILES}

El aspecto que me gustaría citar en este apartado, por su relevancia para buscar una alternativa a esa necesidad socioemocional creada desde el comienzo de la pandemia encontramos a García (2020) aludiendo al reto que se encuentra la escuela marcan el objetivo de no dedicarse solamente a la enseñanza de contenidos y desempeño de asignaturas, sino un seguimiento del aspecto emocional y educativo del alumno, entendiendo que el profesor tiene la tarea encomendada de promover el desarrollo de las competencias sociales y emocionales del alumno, valorando el autocontrol de las emociones, el bienestar personal del alumno y el grupal, teniendo mayor hincapié en la situación extraordinaria que la población mundial está atravesando.

\section{IMPLICACIÓN DEL DESARROLLO SOCIOEMOCIONAL EN SEGUNDO CURSO}

Este apartado describe más específicamente las características del desarrollo infantil enfocado en el primer ciclo de Educación Primaria, atendiendo al curso que nos compete, segundo de primaria, atendiendo el cuidado del desarrollo socioemocional desde el ámbito educativo, explicando los 3 factores que intervienen en este proceso: Inteligencia emocional, Competencia emocional y Educación emocional.

\section{INTELIGENCIA EMOCIONAL}

Se marca un acontecimiento importante en el campo de estudio de la emoción cuando Payne (1986) considera "desarrollo de la inteligencia emocional" palabras clave para el estudio de la emoción y se aplica en el ámbito educativo. Los estudios sobre la inteligencia emocional lideran en Daniel Goleman, quien obtuvo un éxito con su libro "Inteligencia emocional" que publicó en 1995. Goleman asentó sus estudios en las investigaciones de los psicólogos John Mayer y Peter Salovey. Acorde con los estudios sobre la Inteligencia Emocional, FernándezBerrocal \& Ruiz (2008) complementan la definición de Inteligencia Emocional que Salovey y Mayer (1997) basando la Inteligencia Emocional en la solución de los problemas a través de la adaptación de las emociones de manera eficiente. Estos autores establecen cuatro habilidades que forman la Inteligencia Emocional:

Habilidad para percibir, valorar y expresar emociones.

Habilidad para desarrollar sentimientos que facilitan la comprensión al pensamiento.

Habilidad para comprender las emociones.

Habilidad para regular las emociones creando un control intelectual-emocional.

\section{COMPETENCIA EMOCIONAL}

La Competencia Emocional se basa en la fundamentación que sostiene la Inteligencia Emocional, dando lugar al desarrollo de una Educación Emocional. La Inteligencia Emocional abarca un amplio abanico de definición. Bisquerra (2003) propone la Competencia Emocional como el conjunto de conocimientos, capacidades, habilidades y actitudes que se necesitan para entender, expresar y controlar de manera adecuada los componentes emocionales.

\section{EDUCACIÓN EMOCIONAL}

Fernández- Berrocal \& Ruiz (2008) aporta una perspectiva que traslada la Inteligencia Emocional a la educación, denominándola "Educación Emocional" o "Educación Socioemocional" entendiendo este concepto como 
una vertiente que se abre para solucionar los problemas existentes en la educación. Las aportaciones de Trinidad \& Johnson (2002) apuntan que la falta de inteligencia emocional afecta en gran medida a los estudiantes, su ritmo académico y las relaciones sociales, por lo que crean problemas a nivel personal, y en consecuencia a nivel social.

En España se han llevado a cabo programas de educación y se va a referenciar tres de ellos, específicamente, Gómez-Fraguela, Garra \& Romero (2002) proponen "Construyendo Salud" y Segura \& Arcas (2003) plantean el programa "Educación Emocional".

\section{DESARROLLO SOCIOEMOCIONAL EN NIÑOS DE SEGUNDO CURSO DE PRIMARIA}

Desde el estudio de Rodríguez (2005) aporta su perspectiva sobre el concepto de desarrollo socioemocional en los niños de segundo grado de Primaria, estructurado por unas dimensiones que lo forman acompañadas de los indicadores que muestra el niño en torno a esas dimensiones.

Tabla 2. Dimensiones e indicadores que regulan el desarrollo socioemocional. Rodríguez (2005)

\begin{tabular}{|c|c|c|}
\hline Variable & Dimensiones & Indicadores \\
\hline \multirow{4}{*}{$\begin{array}{l}\text { Desarrollo socioemocional: } \\
\text { Rodríguez (2005), el desarrollo } \\
\text { socio afectivo es una dimensión } \\
\text { madurativa del individuo } \\
\text { referida a la formación de los } \\
\text { vínculos con otras personas, al } \\
\text { desarrollo de las emociones y } \\
\text { sentimientos y a la construcción } \\
\text { de una personalidad propia que } \\
\text { incluye motivaciones, intereses, } \\
\text { autoconocimiento. El mundo } \\
\text { afectivo del individuo se genera } \\
\text { de manera interpersonal y } \\
\text { aunque se desarrolló durante } \\
\text { todo el ciclo vital, sus bases se } \\
\text { establecen en la primera } \\
\text { infancia. }\end{array}$} & $\begin{array}{l}\text { La conciencia } \\
\text { social }\end{array}$ & $\begin{array}{ll}\text { - } & \text { Demuestra su ego. } \\
\text { - } & \text { Reconoce su identidad } \\
& \text { personal. } \\
\text { - } & \text { Explica el mundo, la } \\
& \text { naturaleza y la } \\
\text { sociedad. }\end{array}$ \\
\hline & Empatía & $\begin{array}{l}\text { - Se coloca en el lugar } \\
\text { del otro. } \\
\text { - Practica la } \\
\text { comprensión. }\end{array}$ \\
\hline & $\begin{array}{c}\text { Mantener } \\
\text { relaciones positivas }\end{array}$ & $\begin{array}{l}\text { - Rechaza lo malo. } \\
\text { - } \quad \text { Practica lo positivo. }\end{array}$ \\
\hline & Autorregulación & $\begin{array}{l}\text { - Reflexiona sobre su } \\
\text { comportamiento. } \\
\text { - Se autocorrige en } \\
\text { sus emociones. }\end{array}$ \\
\hline
\end{tabular}

Como conclusión a completar el estudio del proceso de desarrollo socioemocional del niño se puede añadir que una regulación en la respuesta socioemocional infantil se encuentra la adquisición de la madurez emocional definida por Erazo Canales \& Salcedo (2018) como la capacidad de aceptar la realidad de las personas y las cosas, tal y como son. La madurez se alcanza cuando las emociones se regulan con racionalidad.

\section{DISEÑO DE LA PROPUESTA DE INTERVENCIÓN}

Para poder aplicar la teoría expuesta anteriormente con situaciones prácticas, he llevado a cabo una propuesta de intervención Ilamada "A distancia". La intervención está dividida en cuatro sesiones repartidas en dos semanas, distribuyendo dos sesiones por semana, que busca como objetivo una alternativa que promueva el desarroIlo socioemocional fomentando la expresión de las emociones y las relaciones sociales del niño en primer ciclo de Educación Primaria, concretamente en el aula de $2^{\circ}$ de Primaria, al mismo tiempo que la calidad de las relaciones sociales en la etapa de pandemia mundial que la población está atravesando. 


\section{OBJETIVOS}

Los 3 objetivos generales que he planteado lograr en el desarrollo del presente trabajo son:

Conocer la expresión de las emociones y el desarrollo de las relaciones sociales del alumnado en segundo curso de Educación Primaria.

Explorar la respuesta socioemocional del alumnado en segundo de Primaria durante la etapa de pandemia de COVID-19.

Buscar nuevas formas de mejorar el desarrollo socioemocional y las relaciones sociales afectadas por la nueva realidad y el aislamiento social provocadas por la pandemia COVID-19.

\section{MUESTRA}

La propuesta se ha llevado a cabo por un grupo de $2^{0}$ curso de Educación Primaria, seleccionados debido a la edad que comprenden, 7 y 8 años. La clase está formada por

24 alumnos, 10 niñas y 14 niños. La decisión de elegir la clase ha seguido los siguientes criterios: Por una parte, siendo sujetos que hayan experimentado desde el comienzo de la etapa de primaria enseñanza presencial antes de la COVID, online durante el confinamiento y presencial actual adaptada a las medidas sanitarias durante la COVID.

Por otro lado, presentación de una actitud participativa y activa durante la realización de dinámicas y actividades.

\section{CRONOGRAMA}

\begin{tabular}{|l|ll|}
\hline SESIÓN 1 & - & $\begin{array}{l}\text { Elaboración cuestionario } \\
-\end{array}$ \\
\hline SESIÓN 2 & - & Visionado de la película (Actividad 2) \\
\hline SESIÓN 3 & - & $\begin{array}{l}\text { Trabajo colorear dibujo de los personajes de la película } \\
\text { (Actividad 3) }\end{array}$ \\
& $-\begin{array}{l}\text { Juego expresión corporal de emociones 'A distancia" } \\
\text { (Actividad 4) }\end{array}$ \\
\hline SESIÓN 4 & $-\begin{array}{l}\text { Dibujo libre expresión de emociones (Actividad 5) } \\
\text { Reelaboración del cuestionario. }\end{array}$ \\
\hline
\end{tabular}

\section{METODOLOGÍA}

Al comienzo de la propuesta de intervención, alumnos consideran debido al porcentaje de respuestas, no haber contemplado hasta ahora otra alternativa que suponga una mejora en el desarrollo socioemocional, y una nueva respuesta socioemocional adaptada a la época de pandemia de COVID-19. La propuesta ha seguido en un primer momento una metodología de aprendizaje basada en el pensamiento (Thinking Based Learning). Los alumnos en la primera parte de la intervención reflexionan sobre las experiencias propias y trabajan individualmente su perspectiva personal de las actividades planteadas. En cambio, en la segunda parte la metodología se convierte en Aprendizaje Cooperativo ya que se desarrolla un trabajo de las actividades propuestas en equipo siendo esencial la colaboración y participación activa del colectivo, desde el respeto entre los compañeros.

\section{INSTRUMENTOS}

La intervención ha comenzado con un cuestionario previo con preguntas relacionadas con la expresión de las emociones y las relaciones sociales desde el comienzo de la COVID-19, que se ha repartido a los alumnos al principio de la primera sesión y me ha permitido investigar las impresiones y pensamientos de los alumnos. Le he atribuido gran importancia a la elaboración de datos a partir de cuestionarios ya que puede obtenerse infor- 
mación de diferentes cuestiones al mismo tiempo. García Ferrando (1993) presenta el cuestionario como una técnica de investigación basada en procedimientos que estudian y justifican un conjunto de características analizando los datos presentados por un número de población. En la aclaración de la definición y finalidad del uso de cuestionarios Sierra Bravo (1994) aporta que el cuestionario es una obtención de datos sociológicos por medio de la interrogación a un número de personas.

Otro de los materiales utilizados para la realización de la intervención han sido los recursos digitales que he utilizado para proyectar la película, las hojas en papel de las diferentes actividades, y por último se ha requerido el uso de pinturas de colores de los alumnos para seguir las instrucciones de las actividades.

\section{RESULTADOS}

La utilización de los cuestionarios, reflejan las respuestas de los 24 alumnos entre 7-8 años de rango de edad, presentes en la propuesta, y de forma gráfica he reflejado una estadística de porcentaje de respuestas atendiendo a la elección de alguna de las preguntas, en concreto la primera en relación con la expresión de las emociones:

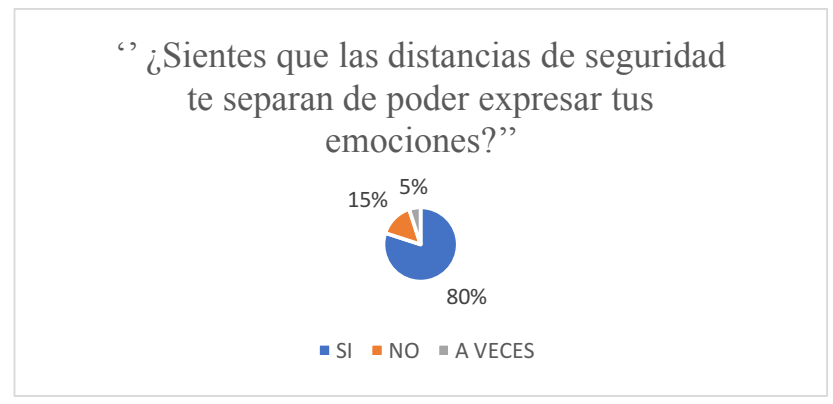

Las respuestas han correspondido en mayor parte a "SI" contando con un $80 \%$ de elección de esa respuesta del total del alumnado, en cambio el 15\% ha respondido "NO", y el 5\% restante ha respondido "A VECES".

Por otro lado, se muestra las respuestas a la siguiente pregunta valorando la calidad de las relaciones sociales:

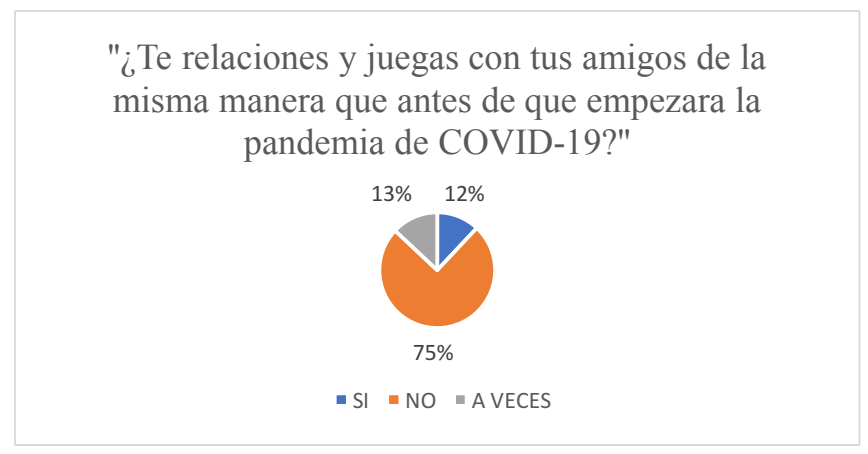

En los resultados se aprecia una clara inclinación al "NO" contando con el 70\%, un 13\% "A VECES" y un $12 \%$ "Sl". 


\section{LA RESPUESTA SOCIO-EMOCIONAL EN TIEMPOS DE PANDEMIA DE COVID 19 EN EL AULA DE SEGUNDO CURSO DE EDUCACION PRIMARIA}

Por último, en cuanto a la muestra de porcentajes de respuestas, se aprecia la pregunta que determina el objetivo de la propuesta de intervención buscando una mejora de la respuesta socioemocional del alumnado, con la siguiente pregunta:

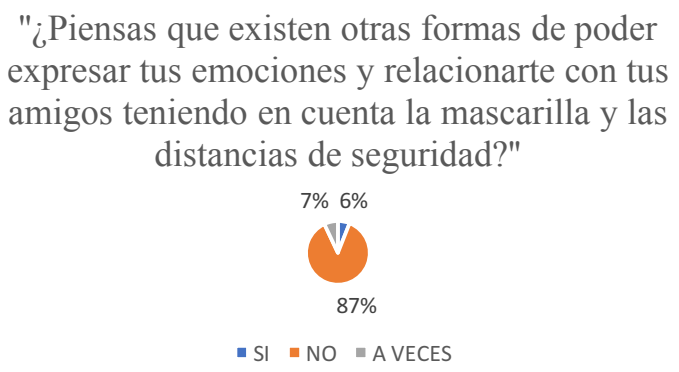

Los resultados e impresiones sobre el desarrollo de la propuesta los he podido establecer entre otros recursos gracias a la repetición del cuestionario. Los alumnos han mostrado una actitud participativa y positiva ante las actividades de la propuesta, aspecto que he considerado esencial para que la intervención se realizara correctamente. Desde mi perspectiva como docente he mantenido una comunicación y prestado la ayuda que los alumnos han necesitado en todo momento. Es importante destacar la inseguridad que han presentado ciertos alumnos al trabajar este tipo de actividades mostrando un desconocimiento en la gran mayoría de los casos total hacia el trabajo del desarrollo socioemocional, dicho en otras palabras, las emociones y el cuidado de las relaciones sociales.

Como es observable, en la repetición del cuestionario se ha apreciado un cambio notable en la respuesta del alumnado cambiando la perspectiva del "NO" al "SI". Este tipo de respuesta ha proporcionado la aseguración del éxito y asentamiento de las actividades por parte de los alumnos. A continuación, se muestra el gráfico representando el cambio de respuesta.

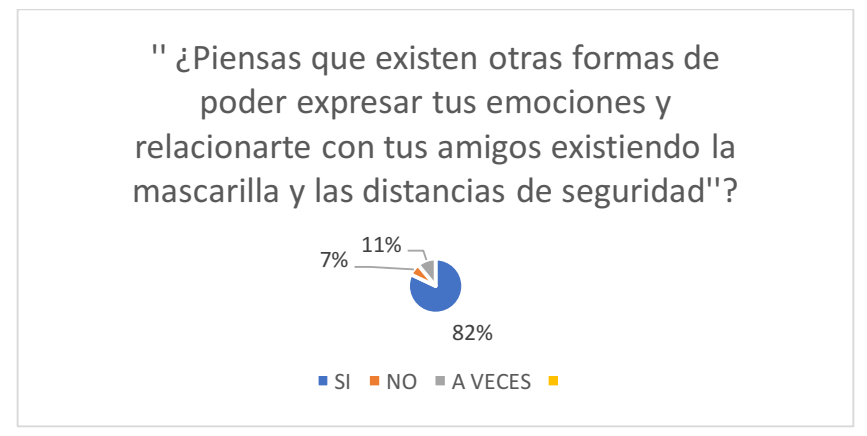

\section{CONCLUSIONES Y FUTURAS LÍNEAS DE INVESTIGACIÓN}

Como conclusión a este trabajo que estudia la respuesta socioemocional infantil en tiempos de pandemia de COVID-19, sitúa al desarrollo socioemocional como un elemento esencial que debe estar vinculado a la educación. 
Por un lado, los objetivos de la siguiente propuesta son conocer y explorar la expresión de las emociones y el desarrollo de las relaciones sociales del alumnado en segundo curso de Educación Primaria, buscando nuevas respuestas socioemocionales del alumnado en segundo de Primaria durante la etapa de pandemia de COVID-19, de manera que a través de esta propuesta de intervención se han llevado estos aspectos a la práctica.

Por otro lado, las limitaciones de este trabajo se han mostrado respuestas de sujetos de género masculino y femenino a través de la práctica de un solo cuestionario por la razón de la edad y la dificultad. Este tipo de pruebas deben adaptarse a la edad de los individuos y el contenido de las preguntas, por ello en este trabajo he realizado un solo cuestionario que esté moldeado al nivel de entendimiento de los alumnos del curso escogido. Asimismo, pueden realizarse variantes en la realización de la propuesta de intervención añadiendo el número de individuos, o incluso dividiéndose por géneros y estudiar los resultados por separado. Así pues, puede centrarse la atención en la variable de niños que son hijos únicos o tienen hermanos, pudiendo observar la calidad de su expresión emocional y de las relaciones sociales. El siguiente paso después de la realización del cuestionario es Ilevar a cabo la propuesta de intervención y, por último, comparar los resultados obtenidos en el cuestionario previo a la intervención y el posterior.

En mi opinión, las emociones forman parte de la evolución del niño desde sus primeros años de vida y mi objetivo como futura docente expresa la labor de atender las necesidades emocionales y sociales del alumnado. Afirmando este argumento, Garaigordobil \& Fagoaga (2006) coinciden en la existencia de acuerdos entre la educación y la psicología, pero pese a la convivencia de las mismas, existe un número reducido de colegios que incluyen experiencias que hacen fusionar estas dos ramas de estudio y como consecuencia el personal docente y psicólogos de los centros educativos, no son formados en la gran mayoría de los casos en programas de intervención que implementen en su metodología el desarrollo socioemocional entre las distintas experiencias a trabajar en el aula. Según Soto \& Bautista (2019) Ios sentimientos de confianza, seguridad, amistad, afecto y humor son esenciales en el proceso de desarrollo socio emocional de un niño, y son aspectos que el docente puede percibir dentro del aula.

A la hora de investigar sobre el impacto que ha tenido la pandemia de COVID-19 en la expresión de las emociones y las relaciones sociales en las aulas de Primaria, Toledo (2020) apunta una necesidad de acceso a herramientas tecnológicas que contemplen el elemento pedagógico en la práctica de esta nueva versión de práctica de aprendizaje, que interpone una barrera virtual en la expresión de las emociones y la pérdida del contacto físico en las relaciones sociales.

Para finalizar, aportando mi perspectiva personal como futura docente, podemos entender el suceso de esta pandemia mundial como un elemento que ha puesto a prueba a la población a sufrir una necesidad de adaptación en la nueva realidad que se vive en la actualidad, y los maestros tenemos como labor estar en un proceso de formación continua que nos prepare para guiar a nuestros alumnos en los nuevos caminos que el traiga el futuro.

\section{REFERENCIAS BIBLIOGRÁFICAS}

Adolphs, R. (2019). Emotions are functional states that cause feelings and behavior. In The Nature of Emotion, Second Edition, A.S. Fox, R.C. Lapate, A.J. Shackman, and R.J. Davidson, eds. (New York: Oxford University Press), pp. 6-10

Adolphs, R., Mlodinow, L. y Barrett, LF (2019). ¿Qué es una emoción? Biología actual, 29 (20), R1060-R1064. (DOCUMENTO)

Alzina, R. B. (2003). Educación emocional y competencias básicas para la vida. Revista de investigación educativa, 21(1), 7-43.

Arellano-Vaca, F. L. (2021). Estrategias docentes para el desarrollo socioemocional en niños con rezago escolar. Bayley, N. (2006). Bayley scales of infant and toddler development. PsychCorp, Pearson.

Bravo, M. P. C. (1994). La metodología cualitativa en España: Aportaciones científicas a la Educación. Bordón. Revista de pedagogía, 46(4), 407-421. 
Erazo Canales, D., \& Salcedo Salcedo, Y. L. (2018). DESARROLLO SOCIOEMOCIONAL EN LOS NIÑOS DEL $2^{\circ}$ GRADO DE LA IEN 23014 ENRIQUE FRACCHIA DE NASCA.

Fernández-Berrocal, P., \& Ruiz, D. (2008). Emotional intelligence in education.

Fernández, J. I. P., Landazabal, M. G., Adrada, Z., \& De Miguel, L. (2011). Efectos de un programa de educación para la convivencia en factores del desarrollo socio-emocional y creativo en niños y niñas de 7 a 11 años. Summa Psicológica UST, 8(2), 5-17.

Ferrando, M. G., Ibáñez, J., \& Alvira, F. (1993). Métodos y técnicas de investigación. Madrid: Alianza Universidad Textos.

Garaigordobil, M., \& Fagoaga, J. M. (2006). Juego cooperativo para prevenir la violencia en los centros educativos: Evaluación de programas de intervención para educación infantil, primaria y secundaria. Madrid: Centro de Publicaciones del Ministerio de Educación y Ciencia.

García-Peñalvo, F. J., Abella-García, V., Corell, A., \& Grande, M. (2020). La evaluación online en la educación superior en tiempos de la COVID-19.

Luengo Martín, M. Á., Gómez Fraguela, X. A., Garra López, A., \& Romero Triñanes, E. (2002). Construyendo salud: Promoción del desarrollo personal y social.

Moreu, Á. C., \& Bisquerra, R. (2002). Los orígenes de la Psicopedagogía: el concepto y el término. REOP-Revista Española de Orientación y Psicopedagogía, 13(1), 17-29.

Munari, A. (1994). Perspectivas: revista trimestral de educación comparada.

Payne, W. L. (1986). A study of emotion: Developing emotional intelligence; Self integration; relating to fear, pain and desire. Dissertation Abstracts International, 47 (01), 2030A (University Microfilms No. AAC 8605928).

Rodríguez, S. (2005). Salud mental del niño de 0 a 12 años. Necesidades básicas del niño.

Salovey, P., y Sluyter, D. J. (1997). Emotional Development and Emotional Intelligence. Educational Implications. Nueva York: Basic Books.

Segura, M., \& Arcas, M. (2003). Mediadores de conflictos cotidianos. Cuadernos de pedagogía, (324), 61-62.

Soto, H., \& Bautista, J. (2019). El Desarrollo Socioemocional y Aprendizaje de estudiantes de tercer grado de nivel Primaria de la Institución Educativa "Revolucionaria Santa Rosa" del distrito de San Sebastián-Cusco 2018.

Toledo, P. A. (2020). Pandemia Covid-19: educación a distancia. 0 las distancias en la educación. Revista Internacional De Educación Para La Justicia Social, 9(3).

Trinidad, D. R., \& Johnson, C. A. (2002). The association between emotional intelligence and early adolescent tobacco and alcohol use. Personality and individual differences, 32(1), 95-105.

Velázquez Pérez, L. (2020). La COVID-19: reto para la ciencia mundial. Anales De La Academia De Ciencias De Cuba, 10(2), e763. Recuperado de http://www.revistaccuba.sld.cu/index.php/revacc/article/view/763/792 